\title{
Biological Activities of a Novel Lectin Derived from Silkworm Faeces: Characteristic Changes of Mouse Peritoneal Macrophages by the Lectin
}

\author{
Etsuko Hirayama, Hisayuki Maekawa, Akihiro Hiraki, and Jeman Kim* \\ Institutes of Molecular and Cellular Biology for Pharmaceutical Sciences, Kyoto Pharmaceutical University, \\ Kyoto 607-8412, Japan
}

macrophages/projections/podosomes/lectin/silkworm faeces

\begin{abstract}
$A B S T R A C T$. A novel lectin derived from silkworm faeces, named NUE, activates phagocytosis of mouse peritoneal macrophages (9). At this time, significant morphological changes of the cells take place. NUEtreated macrophages formed projection-like neurocytes within $12 \mathrm{hr}$ of treatment, and appeared somewhat flat in shape with the activation of cell growth. Associated with the morphological changes, actin was organized in dot-like structures corresponding to cell-substratum contact sites in NUE-treated macrophages. Vinculin, a cytoskeletal protein involved in microfilament-membrane interaction, formed doughnut-like rings matching the actin-dots, called "podosomes". Furthermore, observation by interference reflection microscopy showed that NUE-treated macrophages adhered more strongly to the substratum at local areas. According to these changes, more proteins associated with cell-substratum contact sites became to detergent-resistant. It was shown that NUE changed adhesive form of mouse peritoneal macrophages structurally and qualitatively.
\end{abstract}

Lectins are carbohydrate binding proteins or glycoproteins that have specific affinity for sugar structures (7, 16, 24). Many plant lectins possess immunomodulatory effects. Mitogenic activity on lymphocytes is well known $(10,16)$. Several lectins also have been shown to interact with macrophages. They have been reported to have antitumor activities, that were considered to be due to co-operation with macrophages, such as increase in cell number, release of hydrogen peroxide, and active oxygen radicals $(4,5,13,20,23,25)$. A few reports also have referred to several lectins that stimulate phagocytic activity of macrophages $(6,9,18)$.

Macrophages are highly specialized for phagocytosis. They adhere to a wide variety of foreign bodies, such as glass and plastic. Such adhesiveness is regarded to be one of the initial events of phagocytosis $(1,22$, 27). Their adherence patterns and the distributions of their cytoskeletal molecules during adherence and spreading have been well studied by many authors $(1,2$, $3,15,21,28)$. However, it has not been clarified whether lectins influence the adherence, spreading, or cytoskeletal molecules in macrophages.

A novel lectin isolated from silkworm faeces, NUE, which is a glycoprotein with a molecular mass of about $60 \mathrm{kDa}$, recognizes mannose on cell surfaces and aggregates on many types of cells in suspension (8). Whereas NUE acts as a lectin, it also specifically binds to im-

\footnotetext{
* To whom all correspondence should be addressed.
}

Tel: +81-75-595-4717, Fax: +81-75-595-4798 munoglobulins (Igs) (9). The specific binding to Igs is suggested not to be due to a lectin activity, because NUE can bind to Fab fraction and $L$ chain that do not have sugars. NUE also activates DNA synthesis of lymphocytes and phagocytosis of mouse peritoneal macrophages (9). Thus, NUE is a unique substance with various activities.

When mouse peritoneal macrophages were treated with NUE, we recognized growth activation and remarkable changes in morphology of the cells. Macrophages became flat in shape with the formation of many projections like neurocytes. We observed these morphological changes in more detail by diverse microscopies. In this report, we describe additional activities of the novel lectin, NUE, to macrophages, principally the changes in adhesive patterns according to the morphological changes.

\section{MATERIALS AND METHODS}

\section{Purification of NUE}

NUE was purified from silkworm faeces as described elsewhere (8). The concentration of NUE was determined by the method of Lowry et al. (17).

Cells and cell culture

Mouse peritoneal macrophages were harvested and treated with NUE as previously reported (9). Briefly, $2 \mathrm{ml}$ of $10 \%$ proteose peptone was injected into a mouse intraperitoneally. After 4 days, macrophages were harvested from the peri- 
toneal cavity of decapitated mouse. Harvested cells were seeded at a concentration of $2 \times 10^{6}$ cells $/ 2 \mathrm{ml}$ of minimum essential medium (MEM) containing $10 \%$ calf serum (CS) into a $35 \mathrm{~mm}$-glass dish. After incubation at $37^{\circ} \mathrm{C}$ for $4 \mathrm{hr}$, adhered cells were cultured for another $24 \mathrm{hr}$ at $37^{\circ} \mathrm{C}$. These cells were treated with NUE at a concentration of $200 \mu \mathrm{g} / \mathrm{ml}$ in $10 \%$ CS-MEM as described previously $(8,9)$. This concentration of NUE induces the activation of phagocytosis in mouse peritoneal macrophages as reported previously (9).

\section{Measurement of the growth of macrophages}

Macrophages were treated with $200 \mu \mathrm{g} / \mathrm{ml}$ of NUE for $24 \mathrm{hr}$ and fixed with methanol for $15 \mathrm{~min}$ at $-20^{\circ} \mathrm{C}$. The cells were stained with Giemsa staining solution (Wako Pure Chemical Ltd., Osaka, Japan) for $20 \mathrm{~min}$ at room temperature. After washing by distilled water, the samples were dried. The number of cells in five fields was counted at ramdom, and the average number of the cells in a field and standard deviations were calculated.

When the growth activity was measured by incorporation of $\mathrm{H}^{3}$-thymidine into the cells, $1 \mu \mathrm{Ci} / \mathrm{ml}$ of [methyl $1,2-\mathrm{H}^{3}$ ]thymidine (Amersham Life Sciences, Tokyo, Japan) was added with NUE into the medium, and cultured for $24 \mathrm{hr}$. The cells were solubilized in $0.5 \%$ sodium dodecyl sulfate (SDS), and then equal volumes of $2 \%$ Nonidet P-40 (NP-40) and $10 \%$ trichloroacetic acid (TCA) with SDS solution were added. The mixture was left for $20 \mathrm{~min}$ at $4^{\circ} \mathrm{C}$, the precipitate was harvested onto the glass filter, and washed three times by $5 \%$ TCA. The radioactivity of the precipitate was counted by liquid scintillation counter (LS 5000TA, Beckman, CA, USA). The cells were seeded into $35 \mathrm{~mm}$-glass dishes and incorporation of $\mathrm{H}^{3}$-thymidine per dish was calculated.
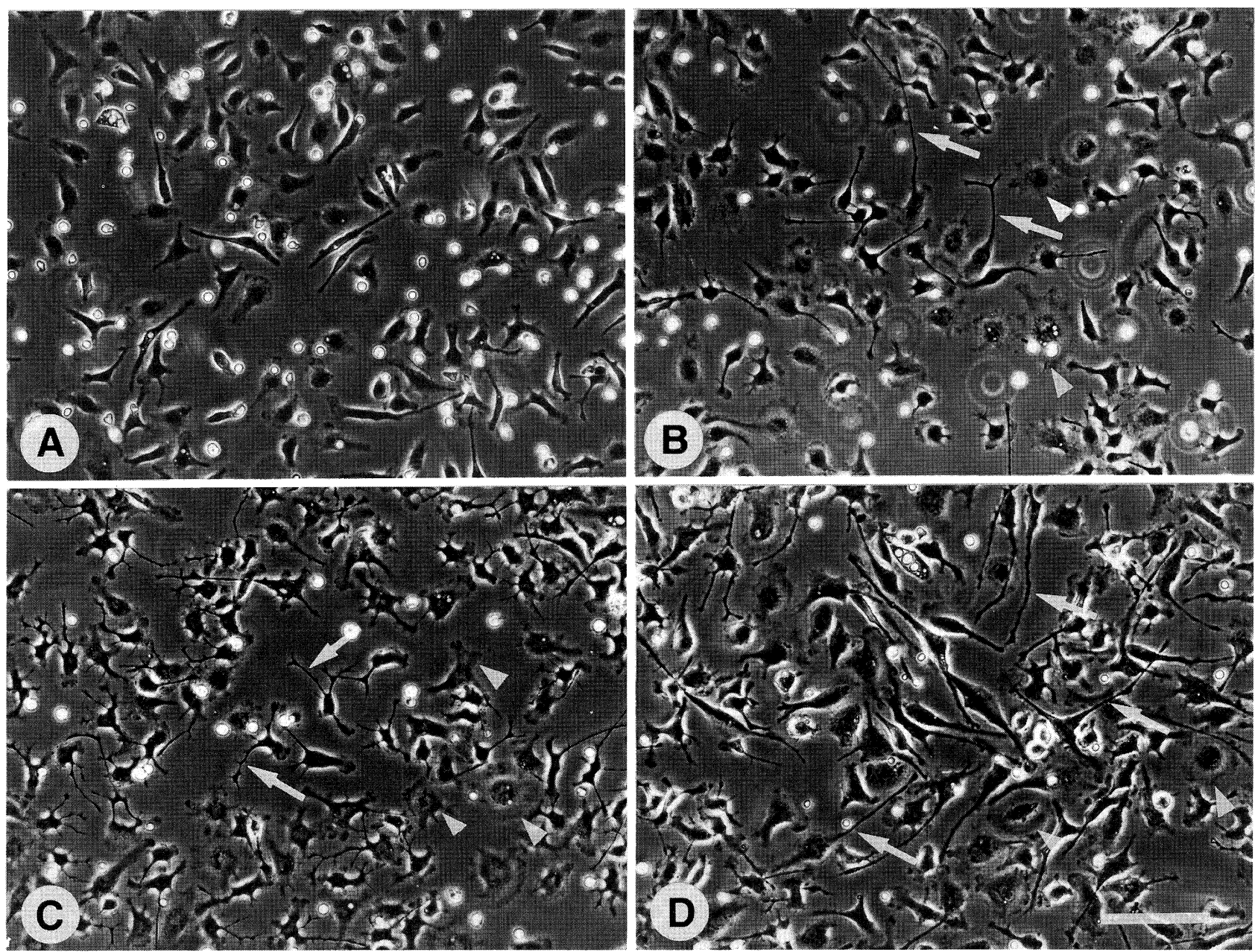

Fig. 1. Morphological changes in NUE-treated macrophages. Mouse peritoneal macrophages were precultured for $24 \mathrm{hr}$ at $37^{\circ} \mathrm{C}(\mathrm{A})$, and the cells were then treated with $200 \mu \mathrm{g} / \mathrm{ml}$ of NUE at $37^{\circ} \mathrm{C}$ for $12 \mathrm{hr}$ (B), $24 \mathrm{hr}$ (C), and $48 \mathrm{hr}$ (D), respectively. After treatment with NUE for $12 \mathrm{hr}$, the cells began to form the projection-like neurocytes (B, arrows). These projections became longer and increased in number with time (C, D arrows), and the cells became somewhat flat in shape and increased in the size (B, C, D, arrowheads). Bar, $100 \mu \mathrm{m}$. 


\section{Indirect immunofluorescent staining}

The detection of F-actin and vinculin was as reported previously (11). Briefly, the cells were fixed in 3.7\% formaldehyde for $30 \mathrm{~min}$ at $4^{\circ} \mathrm{C}$ and treated with $0.5 \%$ Triton X-100 for 5 min at $4^{\circ} \mathrm{C}$. Fixed permeabilized cells were incubated with rhodamine-labeled phalloidin (rho-phalloidin, Molecular Probes, Inc., OR, USA) for staining F-actin and anti-vinculin antibody (a mouse monoclonal antibody against chicken gizzard smooth muscle vinculin, Seikagaku Kogyo Co., Tokyo, Japan), then followed by incubation with fluorescein isothiocyanate (FITC)-conjugated goat antibodies against mouse immunoglobulins $(\mathrm{IgG}+\mathrm{M}+\mathrm{A})$ (Cappel, Organon Teknica, PA, USA). Stained cells were observed with a fluorescent microscope (Axiophoto, Carl Zeiss, Germany) or a confocal laser scanning microscope (LSM 10, Carl Zeiss, Germany). Fluorescent micrographs were taken with Ektachrome 400 (Kodak Co., NY, USA) or TriX-400 (Kodak Co., NY, USA).

\section{Interference reflection microscopy}

For interference reflection microscopy, the cells were fixed in $3.7 \%$ formaldehyde for $30 \mathrm{~min}$ at $4^{\circ} \mathrm{C}$, and observed with a interference reflection microscope (Carl Zeiss, Germany). Micrographs were taken with TriX-400 (Kodak Co., NY, USA).

\section{Electron microscopy}

The cells were prefixed and embedded in Quetol 812 (Nissin EM Co., Tokyo, Japan). The sections were double-stained with uranyl acetate and lead citrate, and observed with a JEOL 2000EXII electron microscope (Nissin EM Co., Tokyo, Japan).

\section{SDS-polyacrylamide gel electrophoresis}

Cells were washed once with PIPES buffer $(10 \mathrm{mM}$ PIPES- $\mathrm{HCl}, \mathrm{pH} 7.0,50 \mathrm{mM} \mathrm{KCl}, 2 \mathrm{mM}$ EGTA, $2 \mathrm{mM}$ $\mathrm{MgCl}_{2}$ ) treated with $0.1 \%$ Triton X-100 in PIPES buffer for 1 min at room temperature, and then washed three times with this buffer. Residual proteins were solubilized with 1\% SDS in PIPES buffer for $60 \mathrm{~min}$ at room temperature and protein concentration was determined by the Lowry method (17).

SDS-polyacrylamide gel electrophoresis (SDS-PAGE) was described by Laemmli (14). Samples ( $1 \mu \mathrm{g}$ of proteins per a lane) were separated in $12.5 \%$ polyacrylamide gel, and proteins were detected by sil-best stain for protein/PAGE, a silver staining kit (Nacalai Tesque, Kyoto, Japan).

\section{Immunoblotting}

Immunoblotting was performed as described elsewhere (12). Anti- $\alpha_{5}$-integrin, $\beta_{1}$-integrin polyclonal antibodies were pur-
A

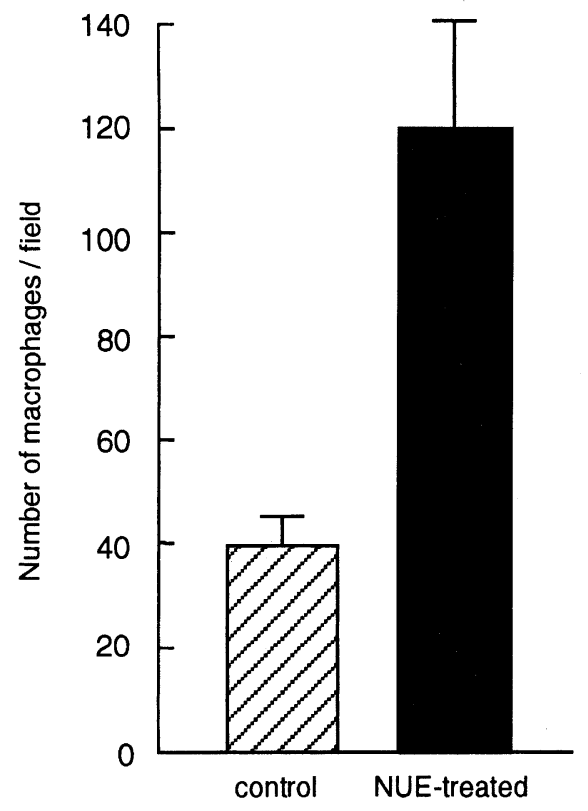

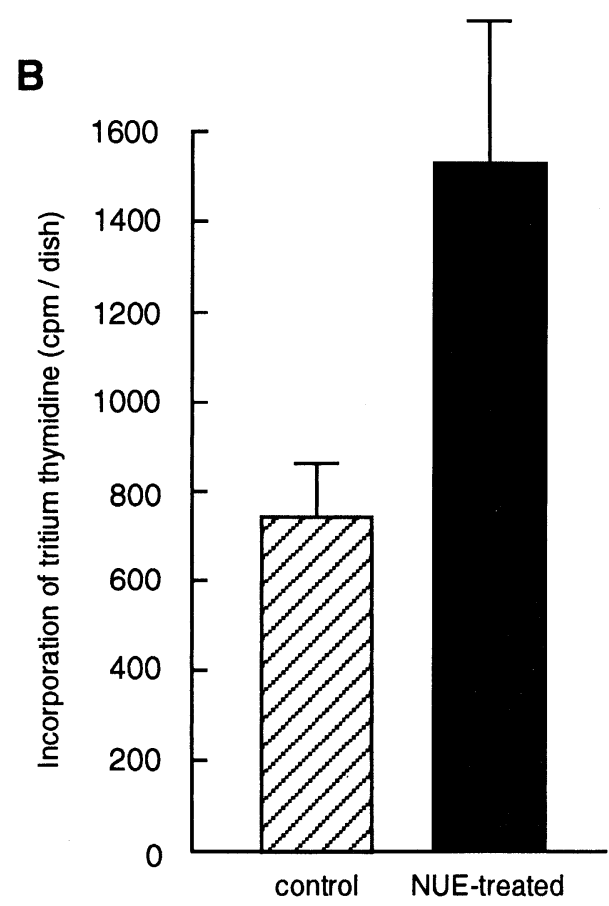

Fig. 2. Growth activation of NUE-treated macrophage. A; Increase of number in a field. Macrophages were treated with or without NUE for $24 \mathrm{hr}$ as in the case of Fig. 1. The cells were fixed and stained with Giemsa staining solution. The numbers of cells in five fields selected at random were counted under optical microscope $(\times 400)$, and the number in a field and standard deviations were calculated. $\square$; the number of NUE-treated macrophages in a field, ש; the number of control cells in a field. B; Increase of incorporation of $H^{3}$-thymidine. In the presence of $\mathrm{H}^{3}$-thymidine, macrophages were treated with NUE and cultured for $24 \mathrm{hr}$. Cells were then solubilized in SDS solution and the incorporation of $\mathrm{H}^{3}$-thymidine was measured by liquid scintillation counter. $\square$; incorporation of $\mathrm{H}^{3}$-thymidine into NUE-treated macrophages, tion of $\mathrm{H}^{3}$-thymidine into control cells, which were not treated with NUE. 
chased from Bioline Dagnostici Co. (Italy). Anti- $\alpha$-actinin monoclonal antibody was purchased from Sigma Chemical Co. (CA, USA).

\section{RESULTS}

Morphological changes in NUE-treated macrophages Mouse intraperitoneal cells were harvested 4 days after injection of proteose peptone and seeded on a glass dish. Macrophages adhered to the dish within $4 \mathrm{hr}$ and showed their typical morphology after $24 \mathrm{hr}$ culture (Fig. 1A). Treatment of these cells with NUE induced characteristic changes in morphology. The projectionlike neurocytes started to form in about $12 \mathrm{hr}$ (Fig. 1B, arrows). These projections could be recognized more distinctly, increased in number with culture time (Fig. 1C, D, arrows). The cells took on a neurocyte-like appearance after culture for $48 \mathrm{hr}$ (Fig. 1D). The cells also appeared somewhat flat compared to the control cells (compare Figs. 1A with B, C, D, arrowheads), and had visibly increased in size. These morphological changes in NUE-treated macrophages did not disappear, even if NUE was removed, suggesting irreversi- ble changes.

Further, such morphological changes were not observed by treatment with other known lectins, concanavalin A (Con A) or wheat germ agglutinin (WGA) (data not shown), suggesting the changes observed in macrophages was specific to NUE.

\section{Growth activation of NUE-treated macrophages}

In the course of the observations shown in Fig. 1, the number of macrophages appeared to increase (compare Figs. 1A with D). To ascertain this, the number of cells was examined after treatment with NUE. Macrophages were treated with NUE for $24 \mathrm{hr}$ and the number of cells in a field was counted under optical microscope. As shown in Fig. 2A, the number was increased as compared with the control. To further ascertain the result, the incorporation of $\mathrm{H}^{3}$-thymidine into the cells was also evaluated (Fig. 2B). The results indicated that NUE treatment activated the growth of macrophages. The growth activation to macrophages was also examined using Con A or WGA. Although both lectins are known to have mitogenic activity to lymphocytes, they did not show the activity against macrophages (data not

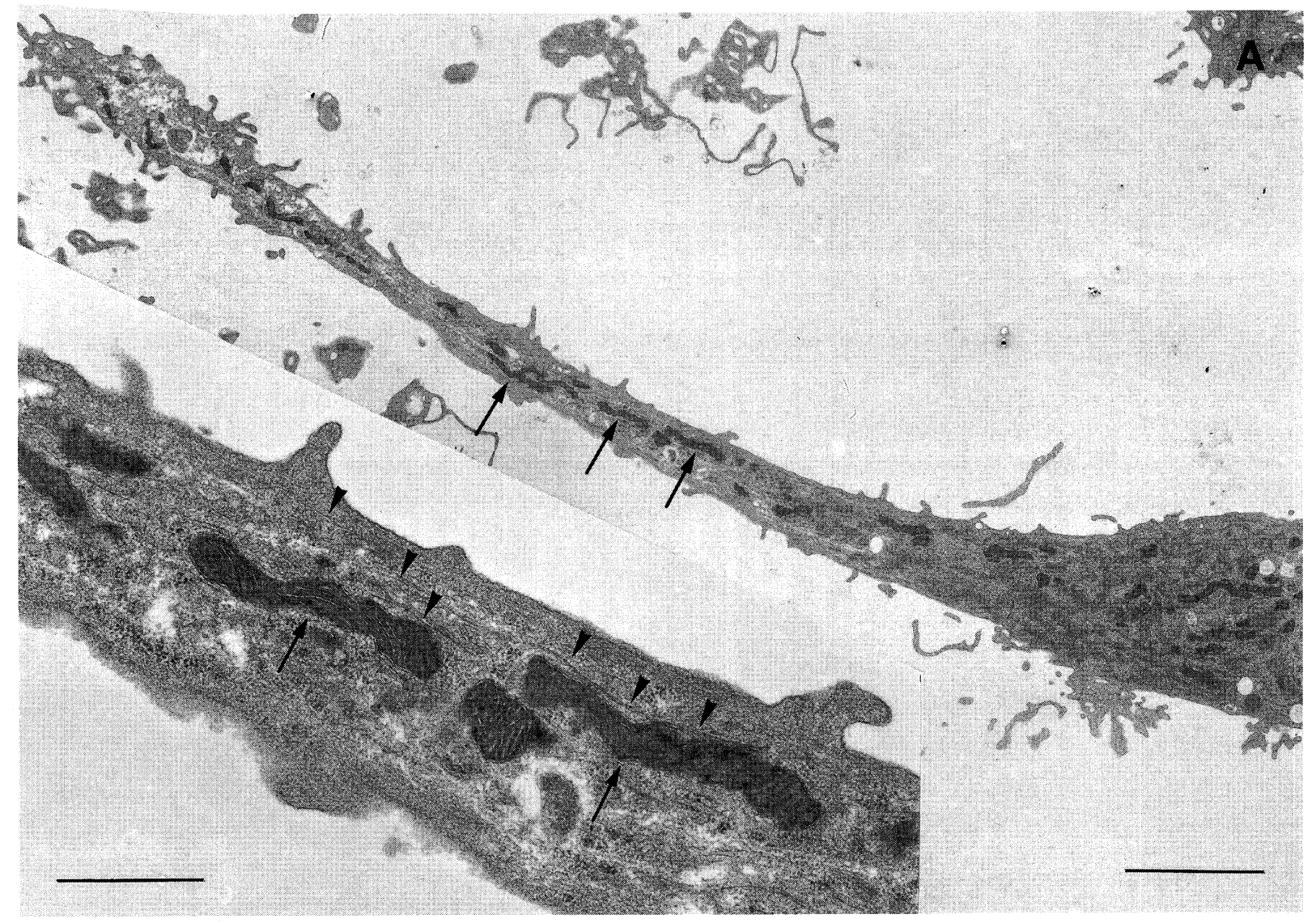

Fig. 3 A 

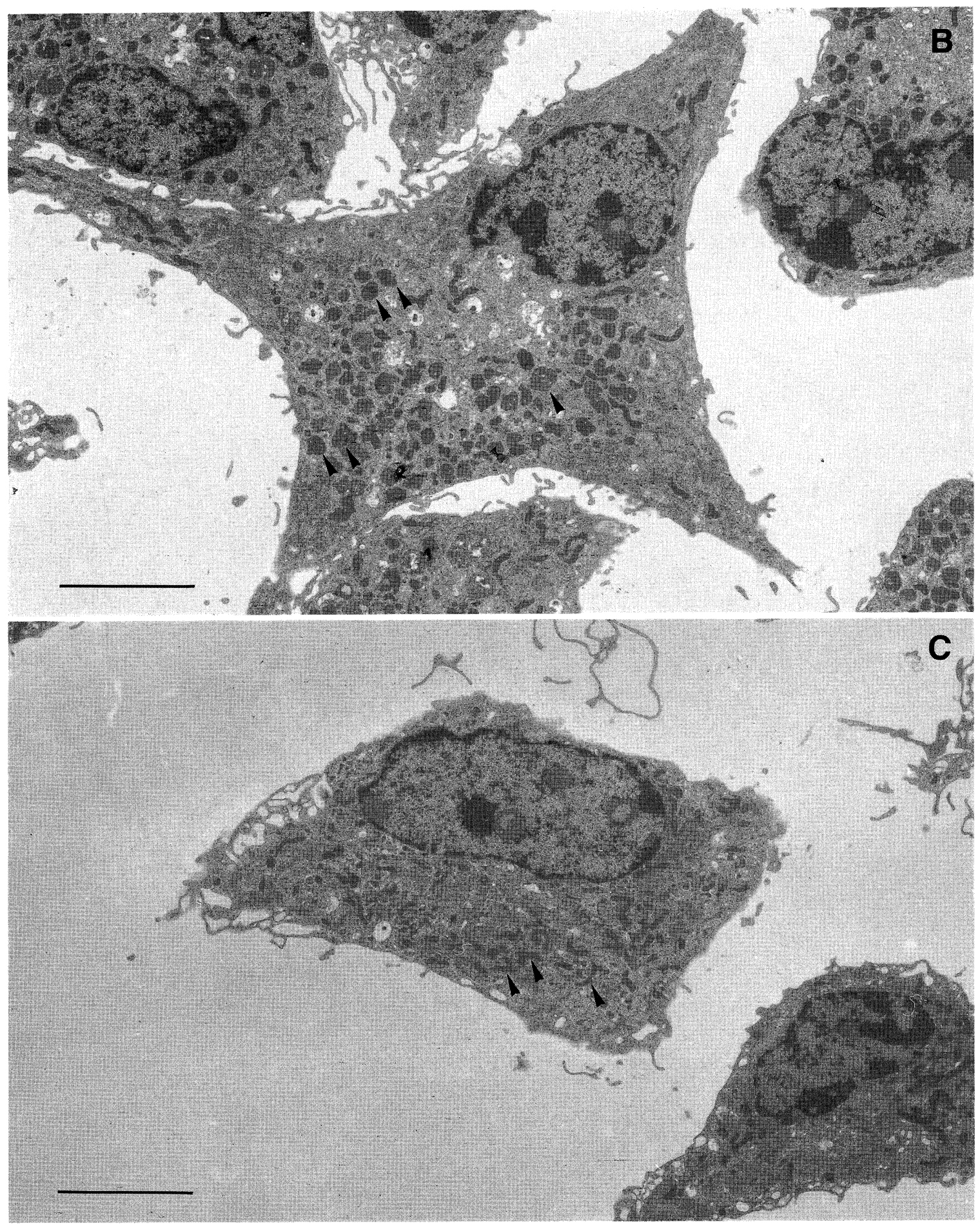

Fig. 3 B, C

Fig. 3. Observations of NUE-treated macrophages by electron microscopy. Macrophages were cultured with or without NUE for $24 \mathrm{hr}$ as in the case of Fig. 1. The cells were fixed and stained for the observation by electron microscopy. A; a projection of NUE-treated macrophages, Bar, $5 \mu \mathrm{m}$, Inset; high magnification of A, Bar, $1 \mu \mathrm{m}$. The projection contained mitochondria (arrow), and was supported by cytoskeletal components elements (arrowheads in inset). B; NUE-treated macrophages, C; control, macrophages cultured without NUE. More round shaped mitochondria were seen in NUE-treated macrophages (compare arrowheads in B with in C). Bars in B and C, $5 \mu \mathrm{m}$. 
shown).

\section{Observation of NUE-treated macrophages by electron microscopy}

To investigate morphological changes of NUE-treated macrophages in more detail, observations by the electron microscopy were performed. Macrophages were treated with NUE for $24 \mathrm{hr}$, and observed by electron microscopy. The projections that formed in NUEtreated macrophages were supported by cytoskeletal filaments such as actin filaments (Fig. 3A, inset, arrowheads) and many mitochondria were distributed to the tip of the projections (Fig. 3A, arrows). On the other hand, control macrophages showed smoother surfaces and projections were few (Fig. 3C). It was recognized that more of the round mitochondria were contained in NUE-treated macrophages (Fig. 3B, arrowheads) than control (Fig. 3C, arrowheads). This observation would suggest swelling of NUE-treated cells.

Macrophages became flat in shape from $12 \mathrm{hr}$-treatment with NUE as described above (Fig. 1B-D). The cells treated with NUE for $24 \mathrm{hr}$ were then sectioned at closer sites to the substratum and observed with electron microscopy. Characteristic microvilli-like structures, that were finer than the projections shown in Fig. 1 or Fig. 3A, were observed in NUE-treated macrophages (Fig. 4A, arrows), whereas such structures were not seen in the control macrophages (Fig. 4B).

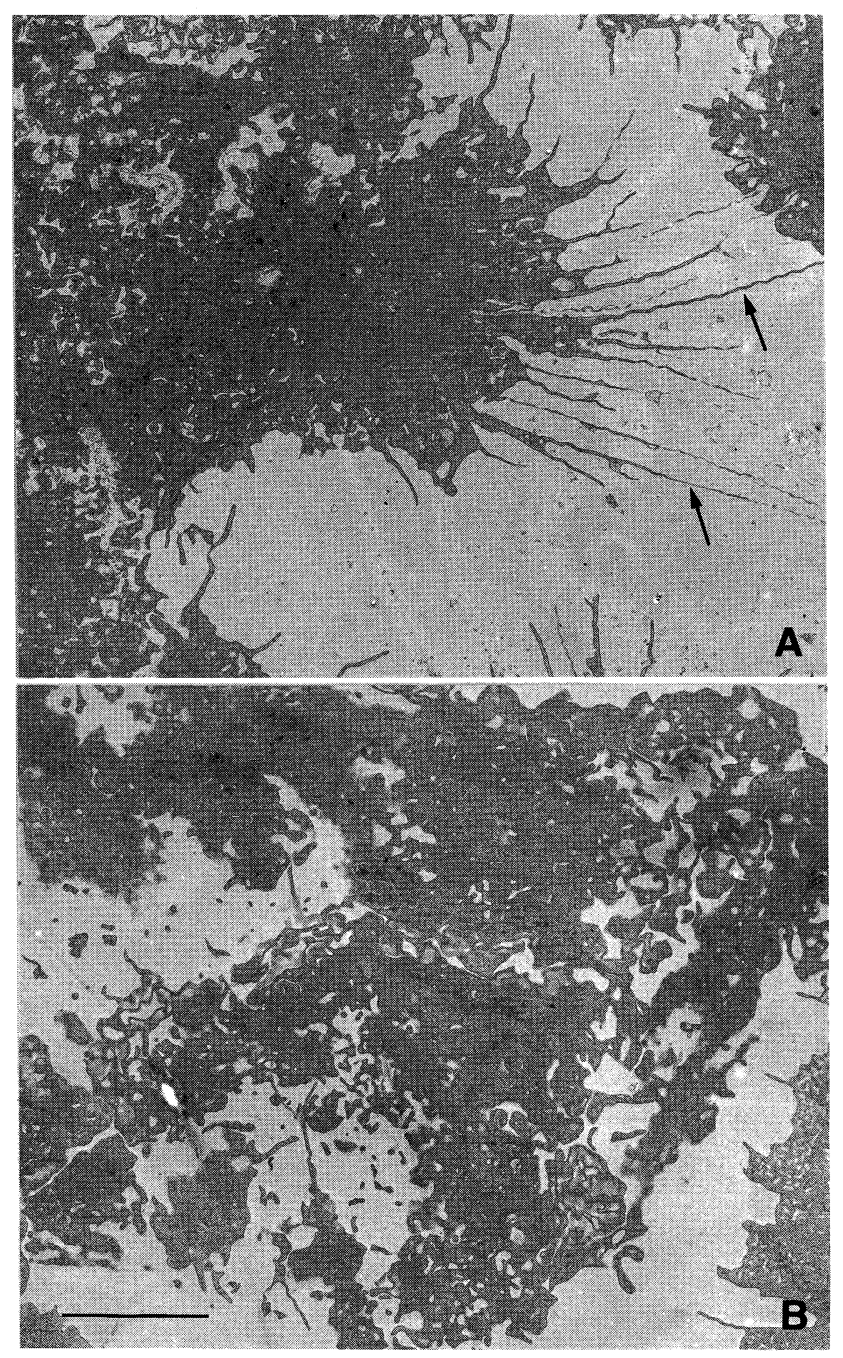

Fig. 4. Characteristic structures near substratum in NUE-treated macrophages. Ultrathin sections in parallel near the substratum of the cells shown in Fig. 3 were prepared, and observed by electron microscopy. A; macrophages treated with NUE, B; control cells, which were not treated with NUE. Many microvilli-like structures were seen in NUE-treated macrophages unlike the control (arrows in A). Bar; $5 \mu \mathrm{m}$. 

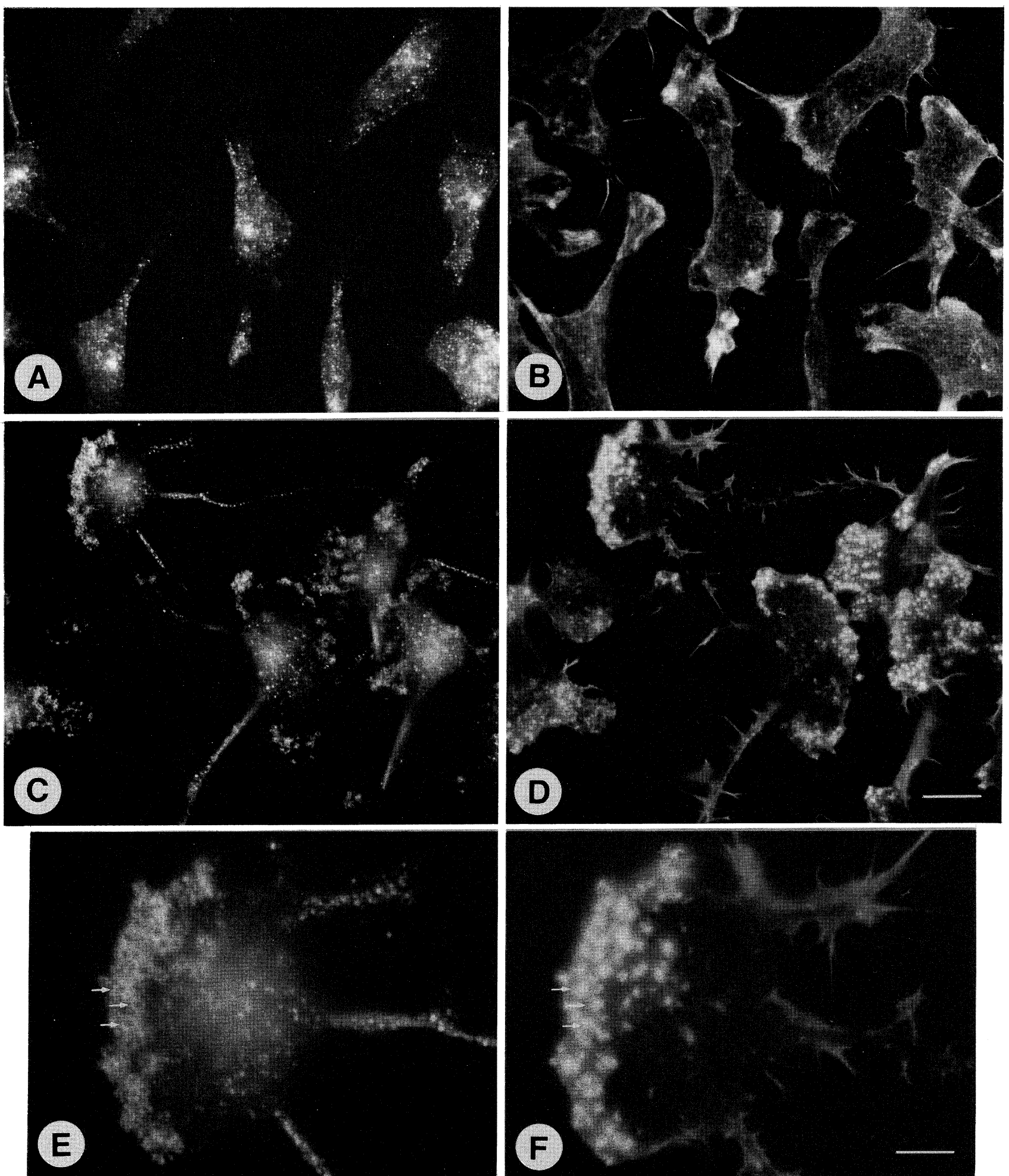

Fig. 5. Changes of distribution patterns of actin and vinculin in NUE-treated macrophages. Macrophages were cultured with or without NUE for $24 \mathrm{hr}$. The cells were fixed in 3.7\% formaldehyde, and then, treated with Triton X-100. Vinculin and actin were double-stained using antivinculin monoclonal antibody $(\mathrm{A}, \mathrm{C}, \mathrm{E})$ and rho-phalloidin $(\mathrm{B}, \mathrm{D}, \mathrm{F}) . \mathrm{A}$ and $\mathrm{B}$ are control macrophages, cultured without NUE. C and D are NUE-treated macrophages for $24 \mathrm{hr}$. E and $\mathrm{F}$ are high magnification micrographs of $\mathrm{C}$ and $\mathrm{D}$, respectively. In NUE-treated macrophages, doughnut-like vinculin-rings were observed matching actin-dots (arrows in E, F). Bar, A, B, C, D; $25 \mu \mathrm{m}, \mathrm{E}, \mathrm{F} ; 10 \mu \mathrm{m}$. 
Changes of distribution patterns of cytoskeletal components in NUE-treated macrophages

It was conjectured that the drastic morphological changes were associated with the changes of distribu- tion of cytoskeletal elements. Thus, we next investigated the distribution patterns of cytoskeletal components in NUE-treated macrophages. Distributions of actin, a member of cytoskeletal filaments, and vincu-
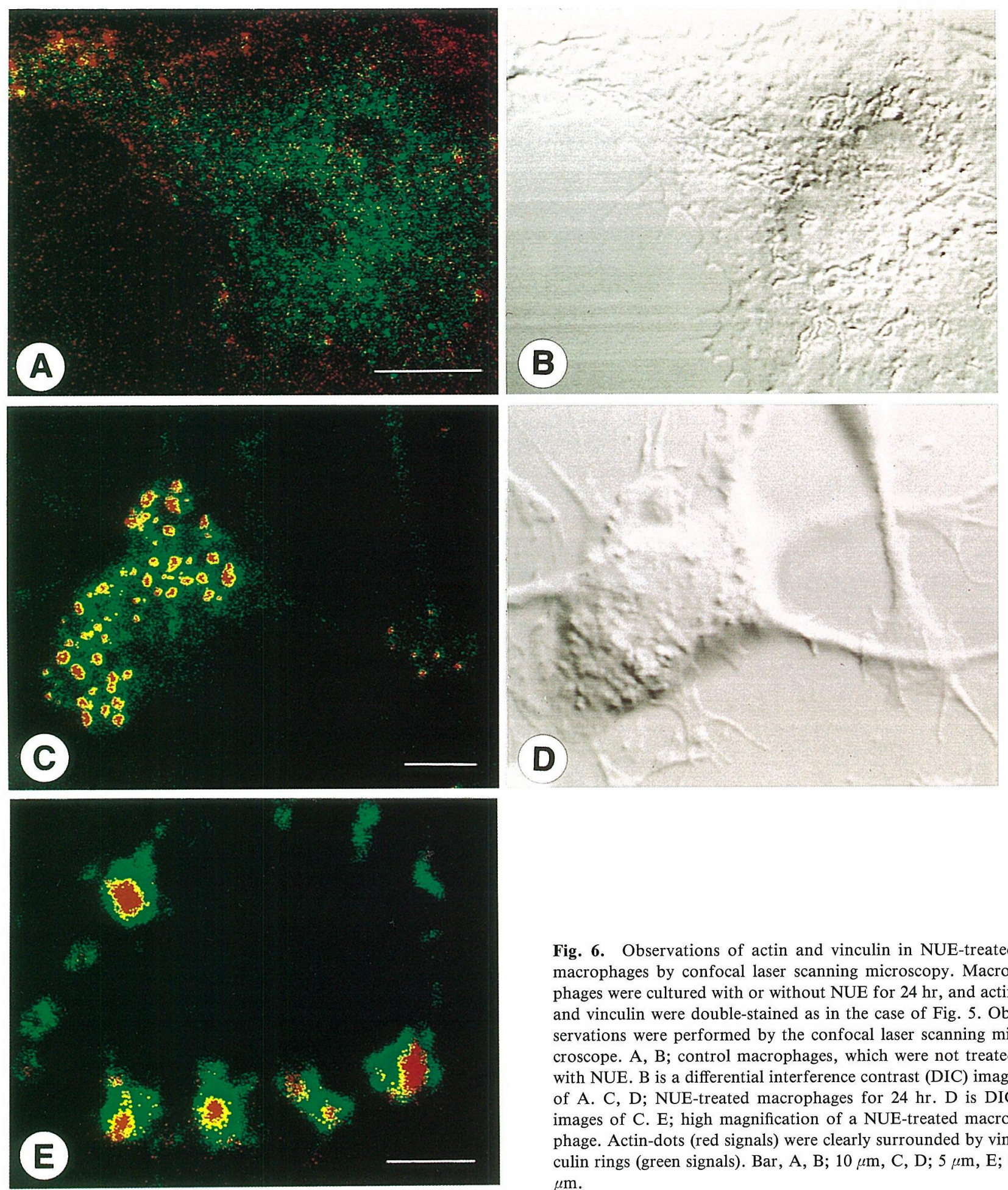

Fig. 6. Observations of actin and vinculin in NUE-treated macrophages by confocal laser scanning microscopy. Macrophages were cultured with or without NUE for $24 \mathrm{hr}$, and actin and vinculin were double-stained as in the case of Fig. 5. Observations were performed by the confocal laser scanning microscope. A, B; control macrophages, which were not treated with NUE. B is a differential interference contrast (DIC) image of A. C, D; NUE-treated macrophages for $24 \mathrm{hr}$. D is DIC images of C. E; high magnification of a NUE-treated macrophage. Actin-dots (red signals) were clearly surrounded by vinculin rings (green signals). Bar, A, B; $10 \mu \mathrm{m}, \mathrm{C}, \mathrm{D} ; 5 \mu \mathrm{m}, \mathrm{E} ; 5$ $\mu \mathrm{m}$. 
lin, which is included in adhesion plaques and connects with actin, were observed by fluorescent microscopy (Fig. 5). In NUE-treated macrophages, peculiar staining patterns were observed. Actin was concentrated in larger dots than adhesion plaques, and doughnut-like vinculin rings surrounded individual actin-dots at contact sites (Fig. 5E, F, arrows). These actin dots were especially evident at the edge of the cells, and seemed to assemble locally. In the control macrophages, such specific patterns were not observed. Diffused or somewhere strongly stained patterns of actin were observed along cell membranes (Fig. 5B). Small dots of vinculin, suggesting adhesion plaques, were observed to be somewhat randomly distributed near the substratum (Fig. 5A).

This characteristic distribution, actin-dots surrounded by vinculin rings, was observed stereoscopically by confocal laser scanning microscopy. In NUEtreated cells, vinculin rings as green signals were detected around concentrated actin dots as red signals (Fig. 6C). At higher magnification, these actin dots were clearly surrounded by doughnut-like vinculin rings (Fig. 6E). On the other hand, in control macrophages (Fig. 6A), specific distribution patterns of actin (red signals) and vinculin (green signals) were not seen. They were detected weakly as diffused signals in the whole of the cell (Fig. 6A). These peculiar structures, actin dots surrounded by vinculin-rings, are possibly adhesion structures called "podosomes" that have already been reported in spreading macrophages, lymphocytes, osteoclast, and transformed fibroblasts $(2,3,15,19,26$, $28,30)$.

\section{Changes of adhesion patterns in NUE-treated macro- phages}

It was shown that NUE-treatment alters the adhesive structures of macrophages. Thus, we next observed NUE-treated macrophages focusing on cell-substratum interaction by interference reflection microscopy, a technique which allowed us to evaluate the distance separating the ventral surface of the cell from the substratum. The results displayed clear differences between control and NUE-treated cells. As shown in Fig. $7 \mathrm{~A}$, cell-substratum adhesion in the control occurred in the broad grey areas as a whole. However, in NUEtreated macrophages, it clearly appeared as the black areas at projections, edge or central area of the cell (Fig. 7B). These black areas coincided with the areas that actin-dots were assembled. Consequently, it was apparent that NUE-treated macrophages adhered more strongly to the substratum than non-treated cells.

\section{Remaining actin structures on substratum after detergent-treatment}

The above results strongly suggested that macrophages

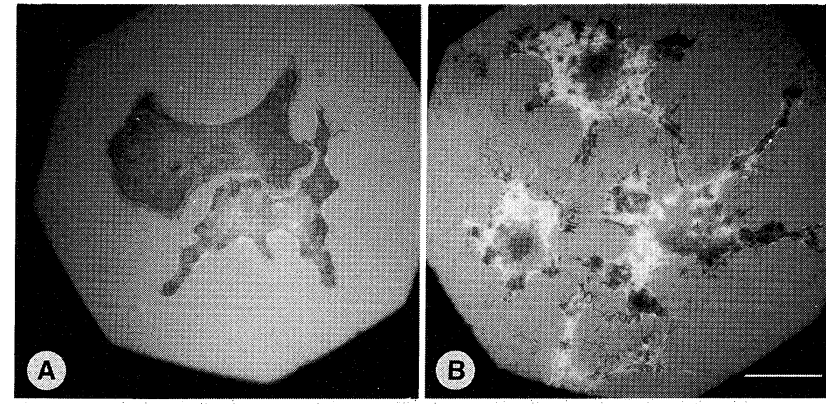

Fig. 7. Observations of NUE-treated macrophages by interference reflection microscopy. Macrophages were cultured with or without NUE for $24 \mathrm{hr}$. The cells were fixed with $3.7 \%$ formaldehyde, and observed by interference reflection microscope. A; control macrophages cultured without NUE, B; NUE-treated macrophages. The black areas were locally seen in NUE-treated macrophages at edges, central, or projections of the cells, suggesting that the cells became more adhesive on treating with NUE. Bar, $20 \mu \mathrm{m}$.

became more adhesive by treatment with NUE. It was conjectured that the result was related to the development of podosomes in NUE-treated cells. To ascertain this point, plated macrophages were removed under the condition of adhesive structures remaining, after exposure to $0.1 \%$ Triton $\mathrm{X}-100$ for $1 \mathrm{~min}$. After exposure to the detergent, almost all the cells were washed out. The residuum on the substratum was fixed and stained with rho-phalloidin for the detection of actin structures in the residuum. In the control macrophages, almost all actin was washed out and a few staining structures were recognized (Fig. 8A). However, in NUE-treated macrophages, many actin structures suggesting podosomes still remained even after the detergent-treatment (Fig. 8B).

Next, the residuum was solubilized in SDS, electrophoresed in SDS-polyacrylamide gel, and proteins were visualized by silver-staining. As a result, more kinds of residual proteins were detected in NUE-treated macrophages than in control (Fig. 9). This suggested that NUE-treated macrophages distributed more proteins in cell-substratum adhesion, to achieve the changes of adhesive form.

It is well known that integrin and $\alpha$-actinin are closely associated with cell-substratum adhesion. Immunoblotting of residual proteins was then performed using anti- $\alpha_{5}$-integrin, $\beta_{1}$-integrin, was detected or $\alpha$-actinin antibodies. In the residuum, only $\alpha_{5}$-integrin detected, and others were not (data not shown).

\section{DISCUSSION}

As reported previouly, we isolated a new lectin, NUE, from silkworm faeces and have investigated its chemical and biological characters $(8,9)$. In the course of in- 

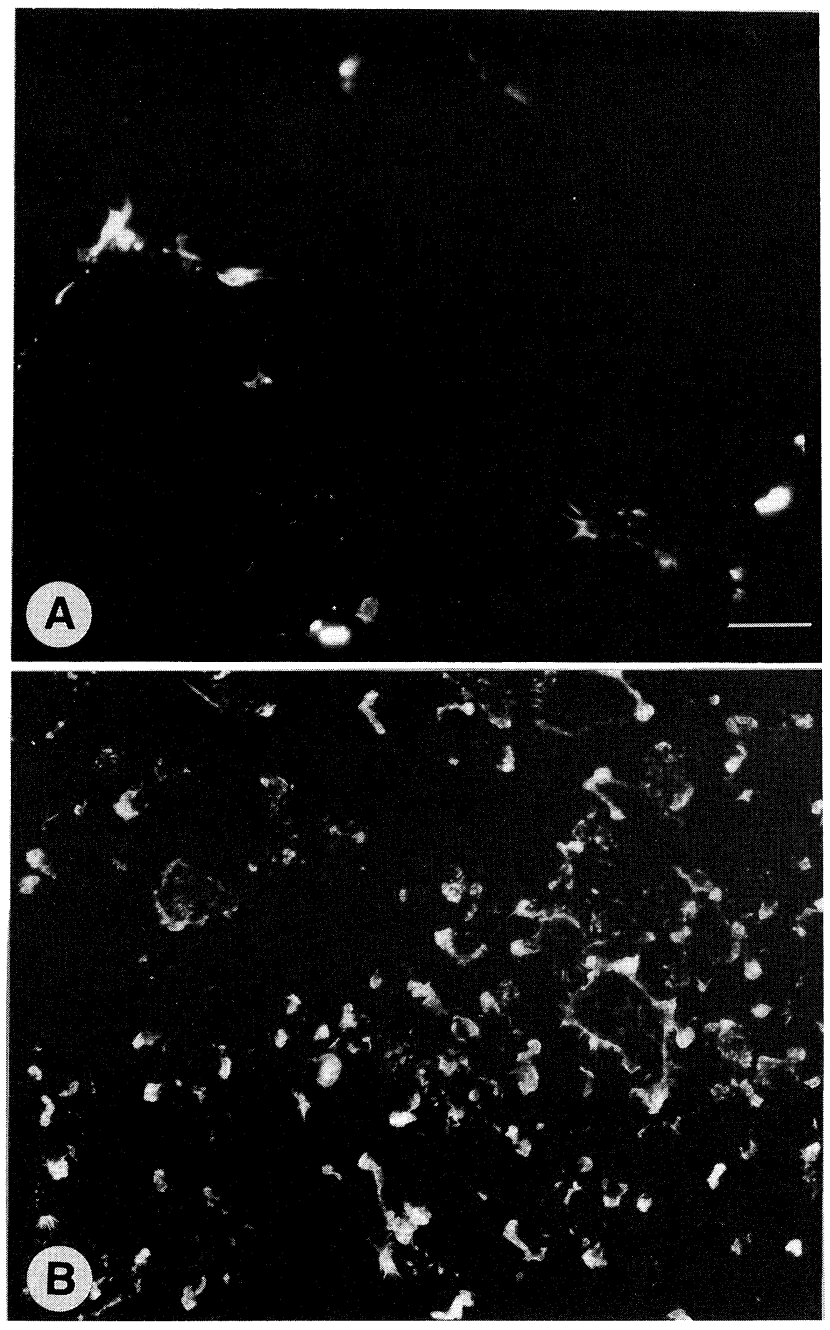

Fig. 8. Remaining of actin structures on substratum after detergenttreatment. Macrophages were treated with or without NUE for $24 \mathrm{hr}$. The cells were then treated with $0.1 \%$ Triton X-100 for $1 \mathrm{~min}$ at room temperature. After washing with PIPES buffer, the residuum was fixed with formaldehyde, and then detected by rho-phalloidin. A; NUE-treated macrophages. B; control macrophages without NUEtreatment. Many actin structures remained even after detergent-treatment in NUE-treated macrophages. Bar, $25 \mu \mathrm{m}$.

vestigation, it was recognized that the morphology of mouse peritoneal macrophages were altered following treatment with the lectin. It has been reported that several lectins increase the number of macrophages (5) and activate phagocytosis $(6,18)$. However, there are few reports that lectins affect their morphology or adhesive patterns. Thus, we examined the morphological changes caused by NUE.

On treatment with NUE, the cells became flat and many projections developed on the cell surfaces. Also many microvilli-like structures were detected near the substratum by electron microscopy. Consequently,

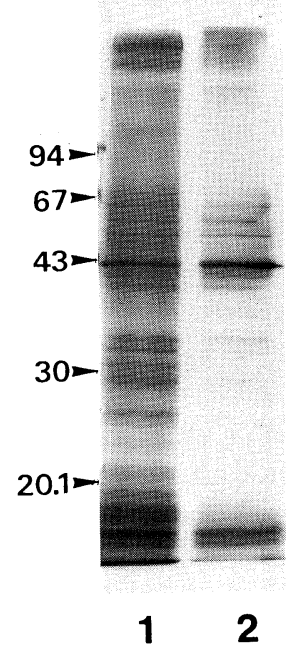

Fig. 9. SDS-PAGE pattern of the residuum on the substratum. NUE-treated, and control macrophages were exposed with Triton X100 as in the case of Fig. 8. Detergent-resistant proteins on the substratum were solubilized in $1 \%$ SDS, electrophoresed in $12.5 \%$ polyacrylamide gel, and then were stained by silver staining. Same amounts of proteins $(1 \mu \mathrm{g})$ were applied to each lane. Lane 1 , detergent-resistant proteins from NUE-treated macrophages, lane 2, detergent-resistant proteins from control macrophages. More kinds of proteins were detected on the dish of NUE-treated macrophages than control cells.

macrophages were changed to neurocyte-like cells in morphology. Along with the morphological changes, the cells developed podosomes on the substratum. As a result, NUE-treated macrophages became more adhesive to the substratum than the control cells. The macrophages thus changed their morphological features. These changes were irreversible; that is, the cells did not return to the former states, even if NUE was removed. Accordingly, a kind of differential reaction may occur on the macrophages by NUE.

When macrophages were treated with other known lectins, Con A or WGA, such morphological, adhesive changes, or growth activation were not seen. Although both NUE and Con A recognize mannose, Con A did not have these activities which NUE had. Furthermore, $\alpha$-methyl-D-mannoside did not inhibit the activities of NUE against macrophages (data not shown), hence the activities in morphology and growth of macrophages are probably not associated with the lectin activity of NUE and is likely to be specific for NUE. Whereas NUE has activities as a lectin, it can bind to immunoglobulins specifically (9). Immunoglobulin (Ig)superfamily plays important roles in many cell functions, such as cell adhesion, recognition, and immunoreaction (29). The activity of NUE that binds to Ig may be related to the changes of macrophages via some Igsuperfamily molecules. 
Adhesion of macrophages to foreign bodies is considered to be one of the event occurring in phagocytosis. Strong adhesion in NUE-treated macrophages may express the activation of phagocytosis reported in the previous report (9). Studies are in progress to clarify the relation between morphological changes and biological functions.

\section{REFERENCES}

1. AgGeler, J. and Werb, Z. 1982. Initial events during phagocytosis by macrophages viewed from outside and inside the cell: membrane-particle interactions and clathrin. J. Cell Biol., 94: 613-623.

2. Amato, P.A., Unanue, E.R., and Taylor, D.L. 1983. Distribution of actin in spreading macrophages: a comparative study on living and fixed cells. J. Cell Biol., 96: 750-761.

3. Boyles, J. and BaInton, D.F. 1979. Changing pattern of plasma membrane-associated filaments during the initial phases of polymorphonuclear leukocyte adherence. J. Cell Biol., 82: 347368.

4. Eckhardt, A.E., Malone, B.N., and Goldstein, I.J. 1982. Inhibition of Ehrlich ascites tumor cell growth by Grifolonia simplicifolia I lectin in vivo. Cancer Res., 42: 2977-2979.

5. Ganguly, C. and Das, S. 1994. Plant lectins as inhibitors of tumor growth and modulators of host immune response. Chemotherapy, 40: 272-278.

6. Goldman, R., Sharon, N., and Lotan, R. 1976. A differential response elicited in macrophages on interaction with lectins. Exp. Cell Res., 99: 408-422.

7. Goldstein, I.J. and HaYes, C.E. 1979. The lectins: carbohydrate binding protein of plants and animals. Adv. Carbohydrate Chem. Biochem., 35: 127-340.

8. Hirayama, E., Ishikawa, N., Yano-Inoue, T., and Kim, J. 1993. Purification and characterization of a lectin-like substance from silkworm faeces. Cell Struct. Funct., 18: 161-171.

9. Hirayama, E., Ishikawa, N., and Kim, J. 1994. Further characterization of a novel lectin derived from silkworm faeces; specific binding to immunoglobulins, and activation of immunocytes. Cell Biol. Inter., 18: 257-269.

10. KilpatricK, D.C. 1995. Lectins in immunology. In Lectins, Biomedical Perspectives (Pusztai, A. and Bardocz, S., eds.). Taylor and Francis Ltd., London, pp.155-182.

11. KIM, J., SAIUCHI, M., and ADACHI, T. 1995. Morphological characters and disturbance of myofibril assembly in avian myoblasts fused with HVJ (Sendai virus). Eur. J. Cell Biol., 66: 94105.

12. Kim, J., Adachi, T., Hirayama, E., Yabubayashi, T., and ОкADA, Y. 1992. Differentiation of quail myoblasts transformed with a temperature sensitive mutant of Rous sarcoma virus. I. Relationship between differentiation and tyrosine kinase of src gene product. Cell Struct. Funct., 17: 237-247.

13. Kurisu, M., YamazaKi, M., and Mizuno, O. 1980. Induction of macrophage-mediated tumor lysis by the lectin wheat germ agglutinin. Cancer Res., 40: 3798-3803.

14. LAEMMLI, U.K. 1970. Cleavage of structural proteins during the assembly of the head of bacteriophage T4. Nature, 227: 680685 .
15. Lehto, V.P., Hovi, T., Vartio, R., Badley, R.A., and VIRTANEN, I. 1982. Reorganization of cytoskeletal and contractile elements during transition of human monocytes into adherent macrophages. Lab. Invest., 47: 391-399.

16. Lis, H. and Sharon, N. 1986. Lectins as molecules and as tools. Annu. Rev. Biochem., 55: 35-37.

17. Lowry, O.H., Rosebrough, N.J., Farr, A.L., and Randall, R.J. 1951. Protein measurement with the Folin phenol reagent. J. Biol. Chem., 193: 265-275.

18. Maldonado, G., Parras, F., Fernándes, L., and Zenteno, E. 1994. Effect of lectins on mouse peritoneal macrophage phagocytic activity. Immu. Inv., 23: 429-436.

19. Marchisio, P.C., Cirillo, D., Teti, A., Zambonin-Zallone, A., and TARONE, G. 1987. Rous sarcoma virus-transformed fibroblasts and cells of monocytic origin display a peculiar dotlike organization of cytoskeletal proteins involved in microfilament-membrane interactions. Exp. Cell Res., 169: 202-214.

20. Nathan, C. and CoHN, Z. 1980. Role of oxygen dependent mechanisms in antibody induced lysis of tumor cells by activated macrophages. J. Exp. Med., 152: 198-208.

21. Ono, M., Murakami, T., Tomita, M., and Ishikawa, H. 1993. Association of the actin cytoskeleton with glass-adherent proteins in mouse peritoneal macrophages. Biol. Cell, 77: 219-230.

22. Reaven, E.P. and Axuine, S.G. 1973. Subplasmalemmal microfilaments and microtubules in resting and phagocytizing cultivated macrophages. J. Cell Biol., 59: 12-27.

23. SAIto, H. and Tomoi, K.H. 1979. Enhanced hydrogen peroxidase release from macrophages stimulated with streptococcal preparation OK432. Infect. Immun., 26: 779-782.

24. Sharon, N. and Lis, H. 1972. Lectins: cell-agglutinating and sugar-specific proteins. Science, 177: 949-959.

25. Shoham, M., Inbar, M., and Sachs, L. 1970. Differential toxicity on normal and transformed cells in vitro and inhibition of tumor development in vivo by concanavalin A. Nature, 227: $1244-1246$.

26. Tarone, G., Cirillo, D., Giancotti, F.G., Comoglio, P.M., and MARChISIO, P.C. 1985. Rous sarcoma virus-transformed fibroblasts adhere primarily at discrete protrusions of the ventral membrane called podosomes. Exp. Cell Res., 159: 141-157.

27. Tomita, M. and IshikawA, H. 1992. Identification of novel adhesion proteins in mouse peritoneal macrophages. Biol. Cell, 76: $103-109$.

28. Trotter, J.A. 1980. The organization of actin in spreading macrophages. The actin-cytoskeleton of peritoneal macrophages is linked to the substratum via transmembrane connections. Exp. Cell Res., 132: 235-248.

29. Williams, A.F. and Barclay, A.N. 1988. The immunoglobulin superfamily-domains for cell suface recognition. Annu. Rev. Immunol., 6: 381-405.

30. Zambonin-Zallone, A., Teti, A., Grano, M., Rubinacci, A., AbBadini, M., Gaboli, M., and Marchisio, P.C. 1989. Immunocytochemical distribution of extracellular matrix receptors in human osteoclasts: A $\beta_{3}$ integrin is colocalized with vinculin and talin in the podosomes of osteoclastoma giant cells. Exp. Cell Res., 182: 645-652.

(Recieved for publication, May 25, 1998 and in revised form, August 4, 1998) 